\title{
How Does a Setting of the Vacuum Energy Density, as Given Today, Lead to an Initial Hubble Radius for the Early Universe, i.e. How Does the Early Universe Partly Mimic a Black Hole?
}

\author{
Andrew Walcott Beckwith \\ Physics Department, Chongqing University, Huxi Campus, Chongqing, China \\ Email: Rwill9955b@gmail.com, abeckwith@uh.edu
}

How to cite this paper: Beckwith, A.W. (2018) How Does a Setting of the Vacuum Energy Density, as Given Today, Lead to an Initial Hubble Radius for the Early Universe, i.e. How Does the Early Universe Partly Mimic a Black Hole? Journal of High Energy Physics, Gravitation and Cosmology, 4, 354-360.

https://doi.org/10.4236/jhepgc.2018.42022

Received: February 28, 2018

Accepted: April 25, 2018

Published: April 28, 2018

Copyright $\odot 2018$ by author and Scientific Research Publishing Inc. This work is licensed under the Creative Commons Attribution International License (CC BY 4.0).

http://creativecommons.org/licenses/by/4.0/

\begin{abstract}
First we review what was done by Klauber, in his quantum field theory calculation of the Vacuum energy density, and in doing so, use, instead of Planck Mass, which has $10^{19} \mathrm{GeV}$, which leads to an answer $10^{122}$ times too large, a cut-off value of instead, a number, $N$, of gravitons, times graviton mass (assumed to be about $10^{-43} \mathrm{GeV}$ ) to get a number, $N$, count of about $10^{31}$ if the vacuum energy is to avoid an overshoot of $10^{122}$, and instead have a vacuum energy $10^{-47} \mathrm{GeV}^{4}$. Afterwards, we use the results of Mueller and Lousto, to compare the number $N$, of $10^{31}$, assumed to be entropy using $\mathrm{Ng}$ 's infinite quantum statistics, to the ratio of the square of (the Hubble (observational) radius over a calculated grid size which we call $a$ ), here, $a \sim a$ minimum time step we call delta $t$, times, the speed of light. Now in doing so, we use a root finder procedure to obtain $\Delta t$ where we use an inflaton value due to use of a scale factor $a \sim a_{\min } t^{\gamma}$ if we furthermore use $\delta g_{t t} \sim a_{\min }^{2} \cdot \phi_{\text {initial }}$ as the variation of the time component of the metric tensor $g_{t t}$ in Pre-Planckian Space-time up to the Planckian space-time initial values.
\end{abstract}

\section{Keywords}

Inflaton Physics, Causal Structure, Non Linear Electrodynamics

\section{Framing the Initial Inquiry}

We will reference what was doing by Klauber [1] as far as a vacuum energy den- 
sity, in his textbook, while replacing the cut-off value he used, that of the Planck Mass, he assumed, with that of the entropy of initial concentration of gravitons, with the presumed mass of a graviton, $10^{-43} \mathrm{GeV}$, in order to come up with a count value of $10^{31}$ for gravitons. Furthermore, if we then next go to Muller and Lousto's entangled entropy [2], we get the 31 times the square of (Event horizon radius/grid size) whereas what we will do is to equate the number, $N$, of about $10^{31}$ with the Muller and Lousto's entropy result, in order to calculate the initial event horizon radius, which we find has a value of about $10^{-20}$ meters. Small enough, according to the comparative calculations, and this depends upon the presumed grid size having a value of $a \sim c$ times $\Delta t$. Here we will reference [3] for how $\Delta t$ is calculated, whereas we will also state that the value of $\Delta t$ is less than or equal to Planck time, [4], which is of the order of 5.39 times $10^{44}$ seconds.

The summary result is that we get a set of conditions for a cosmological version of the event horizon, which is equivalent to stating that the early universe, has some qualitative similarities to a black hole, initially.

In doing this, we are assuming that the entropy as calculated by [2] is in correspondence to the $\mathrm{Ng}$ count of entropy given in [5] which has $S$ (initial) $\sim N$, so in this case we have the number of gravitons, as so calculated being the same as entropy

Finally, after establishing that, we reference a modified version of Hawkings power spectrum for black holes given in Calmert, Car and Winstanley, [6] which is the mechanism of how there is initially a start of leakage through what we claim is a causal barrier, which is a way of having information preservation of Phank's constant, from a prior universe, to today's universe. This means crossing the causal barrier given in [3] so as to preserve information so as to have the same physical law, from cycle to cycle.

In applying [6] we should be alert as to that the expression for $\mathrm{d} E / \mathrm{d} t$ as given, will be having a black body behavior for what is called the absorption cross section, so this absorption cross section is equal to the area of the initial sphere of space-time. In this case we will be looking at the surface area of a bubble of space-time. So this will be markedly different than the black hole case. Otherwise though, much of the construction will be similar.

So, then we will start our inquiry, and to do so, we have the formation of $\Delta t$ so as to create the grid value, $a$, which is in the Muller and Lousto definition of entropy, but notice that we will start the idea of entropy, via an arrow of time argument, [7], i.e. that [2] [5], and [7] will be starting points with equivalent definitions of entropy.

\section{Examination of the Minimum Time Step, in Pre-Planckian Space-Time as a Root of a Polynomial Equation}

We initiate our work, citing [3] to the effect that we have a polynomial equation for the formation of a root finding procedure for $\Delta t$, we can, as we did in [3] do the following. Here this straight from [3]. Copied verbatim, and it will be linked 
to the Muller and Lousto result [2], in the next section afterwards, and then we will right afterwards use [1] for calculating the vacuum energy, with the results of [2] and [3] used so as to explain the significance of what we come up with in our re do of the calculation in [1] with the difference that instead of using the Plank mass in the Vaccum energy calculation, we use $N$ times the mass of a graviton. In doing so we obtain $N \sim 10^{31}$, and if this is a measure of entropy, as by [5] we will then have a way to link the $\mathrm{N}$ of our re done [1] calculation with the Muller and Lousto entangled entropy of [2]. Note that we are below coming up with a precursor of grid size, as in the Muller and Lousto result, [2] which is then compared to how we look at our re do of Equation (1) i.e. through the device of determining a specific grid size, $a$.

So let us now begin to look at the step size for time step, as to get the grid size for [2] first.

$$
\begin{aligned}
& \Delta t \cdot\left|\left(\sqrt{\frac{8 \pi G V_{0}}{\gamma \cdot(3 \gamma-1)}} \cdot \Delta t-1\right)-\frac{\left(\sqrt{\frac{8 \pi G V_{0}}{\gamma \cdot(3 \gamma-1)}} \cdot \Delta t-1\right)^{2}}{2}+\frac{\left(\sqrt{\frac{8 \pi G V_{0}}{\gamma \cdot(3 \gamma-1)}} \cdot \Delta t-1\right)^{3}}{3}-\cdots\right| \\
& \approx\left(\sqrt{\frac{\gamma}{\pi G}}\right)^{-1} \frac{48 \pi \hbar}{a_{\min }^{2} \cdot \Lambda}
\end{aligned}
$$

From here, we then cited, in [5], using [2] a criteria as to formation of entropy, i.e. If $\Lambda$ is an invariant cosmological "constant" and if Equation (1) holds, we can use the existence of nonzero initial entropy as the formation point of an arrow of time [7]

$$
\left.S_{\Lambda}\right|_{\text {Arrow-of-time }}=\pi \cdot\left(\frac{\left.R_{c}\right|_{\text {initial }} \sim c \cdot \Delta t}{l_{\text {Planck }}}\right)^{2} \neq 0
$$

This leads to the following, namely in [5] we make our treatment of the existence of causal structure, as given by writing its emergence as contingent upon having

$$
\left(\frac{\left.R_{c}\right|_{\text {initial }} \sim c \cdot \Delta t}{l_{\text {Planck }}}\right) \sim \vartheta(1)
$$

The rest of this article will be contingent upon making the following assumptions FTR.

In short, our view is that the formation of a minimum time step, if it satisfies Equation (1) is a necessary and sufficient condition for the formation of an arrow of time, at the start of cosmological evolution we have a necessary and sufficient condition for the initiation of an arrow of time. With causal structure, along the lines of Dowker, as in [8] and given more detail by Equation (3) above as inputs into Equations ((1) and (2)) i.e. Planck length is set equal to 1 and by [3] 


$$
\begin{aligned}
& \left.\frac{\Delta E \Delta t}{\text { Volume }} \sim\left[\hbar / \text { Volume } \cdot\left(\delta g_{t t} \sim a_{\text {min }}^{2} \cdot \phi_{\text {initial }}\right)\right]\right|_{\text {Pre-Planckian }} \\
& \left.\underset{\text { (Pre-Planckian }) \rightarrow(\text { Planckian })}{\longrightarrow} \Delta E \Delta t \sim \hbar\right|_{\text {Planckian }}
\end{aligned}
$$

i.e. the regime of where we have the initiation of causal structure, if allowed would be contingent upon the behavior of [2]

$$
\begin{aligned}
& g_{t t} \sim \delta g_{t t} \approx a_{\text {min }}^{2} \phi_{\text {initial }} \ll 1 \\
& \underset{\text { Pre-Planck } \rightarrow \text { Planck }}{\longrightarrow} \delta g_{t t} \approx a_{\text {min }}^{2} \phi_{\text {Planck }} \sim 1 \\
& \left.\Leftrightarrow\left(\frac{\left.R_{c}\right|_{\text {initial }} \sim c \cdot \Delta t}{l_{\text {Planck }}}\right) \sim \vartheta(1)\right|_{\text {Planck }}
\end{aligned}
$$

These conditions are enough to have our creating of $\Delta t$, which is part of how we will come up, with the grid, for the Muller and Lousto calculation of entanglement entropy [2] i.e. we set the grid size, here, to

$$
a \sim c \cdot \Delta t
$$

The equivalence of Equations ((5) and (6)) should be obvious, and we will be using Equation (6) to use [2]'s result next.

\section{Examining of the Muller and Lousto Entanglement Entropy Result, for Early Universe Cosmology}

In short, after a very long set of calculations, their model of entropy comes up with [2], with $r_{H}$ a cosmological event horizon

$$
\begin{aligned}
S(\text { Lousto }) & \sim 0.31 \cdot\left(r_{H} / a\right)^{2} \\
& \sim 0.31 \cdot\left(r_{H} / c \cdot \Delta t\right)^{2} \\
& \sim 0.31 \cdot\left(r_{H} / l_{P}\right)^{2}
\end{aligned}
$$

We argue that this entropy, is equivalent to the count, $N$, of gravitons, in accordance to Ng's infinite quantum statistics, which will then lead to us adapting the next sections results, as to obtain the number of gravitons, initially.

\section{Calcuating the Number of Gravitons, $N$, Due to a Re Set of the Vacuum Energy Calculation Given by Klaubert}

In [1] Klaubert re does the Vacuum energy calculation, using Planck Mass, in order to obtain the following:

$$
\begin{aligned}
& \rho(\text { vacuum-energy-density }) \\
& =\frac{1}{2 \pi} \int_{0}^{\Delta} k^{3} \mathrm{~d} k=\frac{\Delta^{4}}{8 \pi} \\
& \underset{\Delta=\text { planck-mass }}{\longrightarrow} 2.80 \times 10^{74} \mathrm{GeV}^{4}
\end{aligned}
$$

Our supposition is to make the following change in the above calculation, namely 


$$
\begin{aligned}
& \Delta \underset{\text { early-universe }}{\longrightarrow} N \cdot m_{g} \\
& \Rightarrow \rho \text { (vacuum-energy-density) } \\
& =\frac{1}{2 \pi} \int_{0}^{\Delta} k^{3} \mathrm{~d} k=\frac{\left(N \cdot m_{g}\right)^{4}}{8 \pi} \\
& \underset{\Delta=\text { planck-mass }}{\longrightarrow} 10^{-47} \mathrm{GeV}^{4} \\
& \Leftrightarrow N=10^{31} \\
& \& m_{g}=10^{-43} \mathrm{GeV}
\end{aligned}
$$

If, the above $N$, in Equation (9) is the same as entropy, then we can state the following, namely

$$
\begin{aligned}
& N=10^{31} \sim 0.31 \cdot\left(r_{H} / l_{P}\right)^{2} \\
& \Rightarrow r_{H} \sim 10^{15} \times l_{P} \sim 10^{-20} \text { meters }
\end{aligned}
$$

\section{Consequences of Equation (10)}

Here for the satisfying of Equation (10) is contingent upon $\left.R_{c}\right|_{\text {initial }} \sim c \cdot \Delta t$ as of being about Planck Length. Then, we have to deal with inflation. If there are roughly 65 e folds, according to Freeze [9] we have then

$$
R_{\text {initial }} \cdot 10^{27}=R_{\text {final }}
$$

If so, using by [10], the frequency of a wavelength, smaller than $r_{H} \sim 10^{15} \times l_{P} \sim 10^{-20}$ meters will then be on the order of $10^{20}$ Hertz, or about $10^{11}$ $\mathrm{GHz}$, which is then divided by $10^{27}$, which would then indicate a frequency of about $10^{-7}$ Hertz today, which sounds horrendous. But is it clear that the frequency would be of the order of $10^{20}$ Hertz, initially?

Conceivably, depending upon the production scheme, our estimate of $r_{H} \sim 10^{15} \times l_{P} \sim 10^{-20}$ meters depends upon a very specific treatment of an obtained $\left.R_{c}\right|_{\text {initial }} \sim c \cdot \Delta t$ being of the same value as Planck length, and also of $r_{H} \sim 10^{15} \times l_{P} \sim 10^{-20}$ meters being of the order of $10^{-20}$. Also, it depends upon the magnitude of the presumed inflationary expansion i.e. a value of instead of 65 e folds for expansion may instead be 50 e folds of expansion, which would correspondingly dramatically shrink the expansion of the wave function form for gravitational waves.

The only way such questions can be answered, is by experimental inquiry. The above are rough estimates only.

Also the introduction of more analysis from Cosmological Non Linear dynamics, as given in [11] [12] [13] [14] may significantly alter the assumed parameters of our assumptions. Christian Corda, and others have done solid work which may alter what has been presented.

In addition, fidelity, or facts in dispute of the Penrose reference [15] could change the parameter of our assumed problem, greatly again, as well.

The experimental gravity considerations are covered in [16] [17] and [18], and 
the idea should be especially in our work to examine if [16] and [18] in terms of gravity are adhered to. As these are LIGO projects, we should be looking to see if what we are doing contravenes or backs, the post Newtonian approximations of physics, so brought up.

Reference [17] is a must to review. In it, Corda reviews GR tests and our document must not contravene these basics.

We hope our document and its spin offs are in congruence with these last 3 references, and will work on the assumption they are synergistic with respect to our inquiry as stated here.

\section{Acknowledgements}

This work is supported in part by National Nature Science Foundation of China Grant No. 11375279.

\section{References}

[1] Klauber, R. (2013) Student Friendly Quantum Field Theory-Basic Principles \& Quantum Electrodynamics. Santrove Press, Fairfield, Iowa, USA.

[2] Mueller, R. and Lousto, C.O. (1995) Entanglement Entropy in Curved Space-Times with Event Horizons. Physical Review D, 52, 4512-4517.

https://arxiv.org/abs/gr-qc/9504049 https://doi.org/10.1103/PhysRevD.52.4512

[3] Beckwith, A. (2017) How a Minimum Time Step Leads to the Construction of the Arrow of Time and the Formation of Initial Causal Structure in Space-Time. http://vixra.org/abs/1702.0295

[4] Carroll, S. (2004) Space-Time and Geometry: An Introduction to General Relativity. Addison-Wesley, San Francisco, USA.

[5] Ng, Y.J. (2008) Spacetime Foam: From Entropy and Holography to Infinite Statistics and Nonlocality. Entropy, 10, 441-461. https://doi.org/10.3390/e10040441

[6] Calmet, X., Carr, B. and Winstanley, E. (2014) Quantum Black Holes. In: Springer Briefs in Physics, Springer Verlag, New York City, New York, USA. https://doi.org/10.1007/978-3-642-38939-9

[7] Keifer, C. (2012) Can the Arrow of Time Be Understood from Quantum Cosmology? In: Mersini-Houghton, L. and Vaas, R., Eds., The Arrows of Time, A Debate in Cosmology, Springer Verlag, Fundamental Theories in Physics, Vol. 172, Heidelberg, 191-203.

[8] Dowker, F. (2005) Causal Sets and the Deep Structure of Space-Time. https://arxiv.org/abs/gr-qc/0508109

[9] Freeze, K. (1992) Natural Inflation. In: Nath, P. and Reucroft, S., Eds., Particles, Strings and Cosmology, World Scientific, Pte. Ltd., Singapore, 408-424.

[10] Maggiore, M. (2008) Gravitational Waves. In: Theory and Experiments, Vol. 1, Oxford University Press, Oxford, UK.

[11] Camara, C.S., de Garcia Maia, M.R., Carvalho, J.C. and Lima, J.A.S. (2004) Nonsingular FRW Cosmology and Non Linear Dynamics. Version 1.

Arxiv astro-ph/0402311

[12] Corda, C., Herman, J. and Cuesta, M. (2011) Inflation from $R^{2}$ Gravity: A New Approach Using Nonlinear Electrodynamics. Astroparticle Physics, 34, 587-590. 
https://doi.org/10.1016/j.astropartphys.2010.12.002

[13] De Lorenci, V.A., Klippert, R., Novello, M. and Salim, J.M. (2002) Nonlinear Electrodynamics and FRW Cosmology. Physical Review D, 65, 063501. https://doi.org/10.1103/PhysRevD.65.063501

[14] Corda, C., Herman, J. and Cuesta, M. (2010) Removing Black-Hole Singularities with Nonlinear Electrodynamics. Modern Physics Letters A, 25, 2423-2429.

https://arxiv.org/abs/0905.3298 https://doi.org/10.1142/S0217732310033633

[15] Penrose, R. (1965) Gravitational Collapse and Space-Time Singularities. Physical Review Letters, 14, 57. https://doi.org/10.1103/PhysRevLett.14.57

[16] Abbott, B.P., et al. (2016) Observation of Gravitational Waves from a Binary Black Hole Merger. Physical Review Letters, 116, 061102.

https://doi.org/10.1103/PhysRevLett.116.061102 https://physics.aps.org/featured-article-pdf/10.1103/PhysRevLett.116.061102

[17] Corda, C. (2009) Interferometric Detection of Gravitational Waves: The Definitive Test for General Relativity. International Journal of Modern Physics D, 18, 2275-2282. ArXiv: 0905.2502 [gr-qc] https://doi.org/10.1142/S0218271809015904

[18] Abbot, B.P., et.al. (2016) GW151226: Observation of Gravitational Waves from a 22-Solar-Mass Binary Black Hole Coalescence. Physical Review Letters, 116, Article ID: 241103. https://doi.org/10.1103/PhysRevLett.116.241103 